\title{
Research on the Structure of Linear Oscillation Motor and the Corresponding Applications on Piston Type Refrigeration Compressor
}

\author{
Wang Ya Tao ${ }^{1, a}$, \\ ${ }^{1}$ TianJin Maritime College , \\ TianJin , 300350 China
}

\author{
Wu Fei ${ }^{1, a}$ \\ ${ }^{1}$ TianJin Maritime College , \\ TianJin , 300350 China
}

\begin{abstract}
In this paper, we conduct research on the structure of linear oscillation motor and the applications on piston type refrigeration compressor. Linear compressor compared with traditional piston compressor mainly has the advantages of energy saving, low noise and easy control. Piston type refrigeration compressor and screw refrigeration compressor are using volume reduction to improve cooling steam pressure, so as to achieve the purpose of refrigeration. Our research analyzes the advantages of the linear oscillation motor and its applications to the real world mechanical devices which will help the industry realize the technique better.
\end{abstract}

Keywords: Refrigeration Compressor; Piston Type; Linear Oscillation Motor; Space Structure.

\section{Introduction}

Compressor is the drive components compression refrigeration system, it is very important for the performance of the refrigeration system efficiency, adopt efficient refrigeration compressor, can significantly improve the performance of refrigeration coefficient and can effect comparing. Linear oscillation motor is a kind of short stroke caused by electromagnetic force directly, high frequency reciprocating motion of the motor. Relative to the rotating machine and the crank shaft produce linear motion, an indirect method, linear oscillation motor saves intermediate conversion mechanism, the characteristics of high transmission efficiency, compact structure and the advantages of small noise, very suitable for artificial heart, pump and compressor, etc. Moving magnet for dynamic quality is lighter, good dynamic characteristics and high efficiency which has been more widely used. Traditional linear reciprocating motion usually adopts rotating motor in combination with the crank transmission mechanism. This way has some shortcomings, such as low speed vibration and noise, big size and not easy to achieve no oil, etc. Linear compressor compared with the traditional piston compressor mainly has the advantages of energy saving, low noise and easy control. Due to eliminating the crank transmission mechanism and reducing the pressure on the piston to the compression chamber wall, the loss system would be more efficient. Move the child directly with the compressor piston rigid connection, drives the piston reciprocating linear motion, made integrated linear oscillating compressor system, can improve the space utilization and reduce compressor system volume which will improve the power density [1]. Piston stroke can be quickly adjusted according to the need of the refrigerating capacity, eliminating the conversion mechanism, improve the efficiency of the system is running, so the refrigerator industry has great application prospect.

Piston type refrigeration compressor at home and abroad, has a long history, as early as 100 years ago the world had been put into use, through continuous improvement, the increasing maturity of the technology, the product has high technical and economic indexes, it is generally believed the technology to improve degree has reached 95 96\%. We know that high pressure steam condensation temperature is higher, so the 
refrigerant vapor as long as the compressor compressed into the condensing temperature corresponding to the saturation pressure which can use the environment medium to cool, make the heat during the cooling object and gasification of refrigerant vapor to condense into liquid, and then let the refrigerant liquid at low temperature and heat gasification, such refrigerants in a closed system, consumption of the compressor work can only repeatedly to realize refrigerant change from liquid to vapor, again by vapor phase change of liquid, and the transfer of heat by the low temperature phase change will be to high temperature. This is the working principle of vapor compression machine [2]. Piston type refrigeration compressor and screw refrigeration compressor are using volume reduction to improve cooling steam pressure, so as to achieve the purpose of refrigeration, so both the volume type compressor and they have the different characteristics, in the refrigeration compressor has an absolute advantage in the market. In the following figure one, we illustrate the general organization and structure of the compressor.

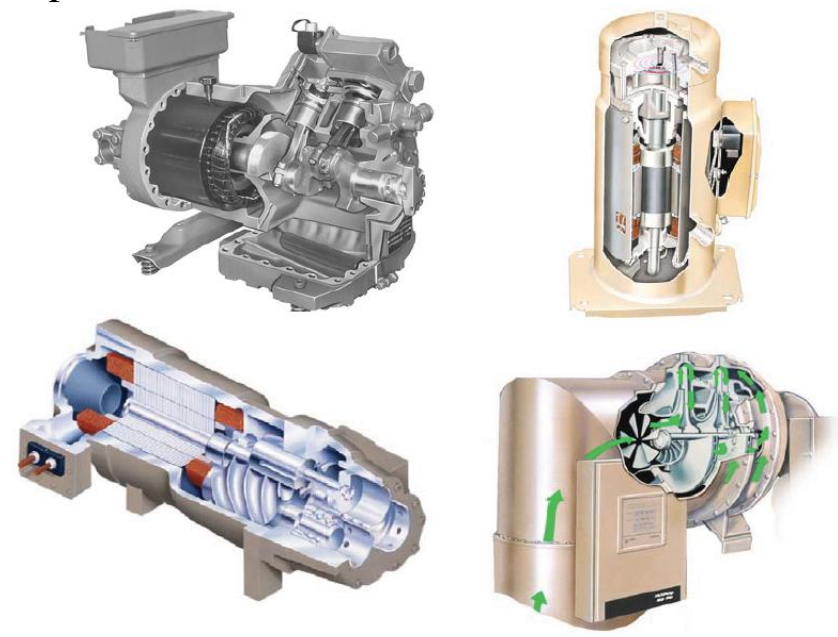

Figure 1.The General Organization and Structure of the Compressor

In this paper, we conduct research on the structure of linear oscillation motor and the applications on piston type refrigeration compressor. Improve the enclosed piston type refrigeration compressor can effect comparing is the most important way to improve the efficiency of the motor. The traditional refrigerator compressor motor is single-phase induction motor, we use high magnetic induction low silicon steel sheet metal loss, and uses the annealing process and optimize the design of the motor can greatly improve the efficiency of motor, but still cannot meet the needs of the efficient refrigerator compressor. The next paragraphs will introduce our proposed novel methodology.

\section{Our Proposed Methodology}

The Piston Type Refrigeration Compressor. There are many types of the piston type refrigeration compressor structure style, according to the different way of the combination of the compressor and the motor can be divided into the open, semi-closed and totally enclosed three kinds. Open is separated from the compressor and motor, compressor crankshaft has end out of the body, and connected by coupling and motor. Semi-hermetic compressor is the compressor and motor assembly together in a detachable sealed inside the casing, the compressor crankshaft and motor rotor shaft is a whole shaft, compressor without moving parts are out of the body. Totally enclosed compressor is the compressor and motor assembly together within a closed casing, casing of welded joints with welding method. The piston type refrigeration system performance characteristics could be summarized as the follows. (1) Small volume and covers an area of less. The unit for semi-closed piston compressor, evaporator with low noise fan. Refrigeration system without frozen water cycle system, and the cooling pipe system of pipe diameter is smaller. Normal noise comes from the compressor and the fan in the system, in the installation of the compressor under the condition of reasonable, with low noise fan, indoor noise can be controlled within $70 \mathrm{db}$. Cooling system adopts the water tower condenser circulating cooling water, the water tower is located in the roof of the workshop, is a high 
efficiency packing type water tower, the water temperature can guarantee as close to the wet bulb temperature. (2) Piston compressor piston from cylinder head start, the work within the cylinder volume increases gradually, at this time, the gas along the inlet pipe, pushed open the inlet valve into the cylinder, until the work gets to maximum volume, the inlet valve close. Reverse movement, piston compressor piston within the cylinder working volume shrinking, gas pressure, when pressure in the cylinder reaches and slightly higher than the exhaust pressure, exhaust valve opens. Gas discharge cylinder, until the piston movement to the limit position, exhaust valve closed. (3) Single-phase ac permanent magnet synchronous motor stator structure and trough with single-phase ac induction motor is the same, but different rotor slot form and structure. Single-phase ac induction motor for squirrel cage rotor is composed of iron core and aluminum cage. When the load changes, single-phase ac induction motor air gap resultant magnetic field changes over load, so that the motor speed increased with the increase of load. Single-phase ac permanent magnet synchronous motor speed is only related to the power frequency and the number of motor, when the motor load changes, the bilateral excitation characteristics will quickly gap resultant magnetic field with load change to ensure the constant.

The Linear Oscillation Motor. Linear oscillation motor automatically generated high frequency reciprocating linear motion, characteristics of synchronous, induction, a variety of types such as step by step, used in refrigeration compressor system mainly for the single-phase permanent magnet synchronous linear oscillation motor, according to the dynamic of different materials, can be divided into the following several kinds of structure. (1) Moving coil type permanent magnet linear oscillation motor. Coil winding of motor parts as a movement, and spring together, the established permanent magnet excitation winding in the stator magnetic field, when the sinusoidal alternating current coil, would have a positive and negative minimization algorithm on moving the son of electromagnetic thrust, there is no radial force on the moving coil, has a simple structure, convenient control, low cost advantages, and the moving coil type permanent magnet linear electric motor quality is lighter, so high resonance frequency, low starting current, low energy loss. But as a result of moving parts for coil, coil wiring place easily broken, commonly used in small power short stroke. (2) Moving magnet type permanent magnet linear oscillation motor. Working magnetic field generated by the stator coil of the armature magnetic field and the dynamic excitation magnetic field permanent magnet constitute a son. Under the interaction of two magnetic field and produce axial electromagnetic driving force to promote child do linear motion. Such a structure will move easily made permanent magnets embedded structure, is advantageous to the protection of permanent magnet, and the force characteristic is better. But the child still lays particular stress on quality, low resonant frequency. (3) Moving magnet type permanent magnet linear oscillation motor. Vibration part is only installed on the permanent magnet on magnetic stent, quality is light, and in as part of the stator iron core and outer core. The air-gap magnetic field contains constant excitation magnetic field produced by permanent magnet and the coil into the current produced by alternating the armature magnetic field. Under the interaction of two magnetic fields and the axial electromagnetic force to promote do reciprocating linear motion. This kind of structure is more compact, dynamic quality relatively light, and thrust density is larger and the resonance frequency of a structure [3].

Core and core, spring together, using magnetic resistance as a electric motor drive. When coil into the current, the armature magnetic field respectively for parallel arrangement of opposite polarity have increased magnetic weak magnetic effect of permanent 
magnet, so as to make the moving core to increase magnetic lateral movement. When alternating current, can produce alternating magnetic resistance of the corresponding thrust to promote child do reciprocating linear motion, move easily from the air gap center axis to produce larger radial force. Moving iron type linear oscillation motor electromagnetic air gap is smaller, so the force is bigger. Double stator moving magnet linear oscillation motor inside and outside fold in process the same as the rotary motor, stator core domestic is relatively mature; LG moving magnet linear oscillation motor stator iron core stack with complex, such as inner stator core silicon steel sheet along the circumference of a circle to be loaded into the exterior inside circle columnar. LG moving magnet structure, winding demagnetization exert on the same piece of permanent magnets, magnetic potential and double stator moving magnet and moving magnet type is applied on the two pieces of permanent magnet.

Dual structure of the linear oscillation motor stator resistance to demagnetization ability is higher than LG moving magnet structure. For dynamic quality, double stator moving magnet structure for permanent magnet with LG moving magnet structure and quality of lighter stents, and therefore easy to realize the high frequency large amplitude oscillations. Double stator moving magnet type structure contained in the part of the silicon steel sheet pile as a core, so the quantity increase, not easy to achieve high frequency oscillations. Outside of the permanent magnet on both ends of the air gap only armature magnetic field caused by reverse current, the stator gap only permanent magnet, and for no ferromagnetic air boundary, and outside of the stator and permanent magnet chain within the scope of the air-gap magnetic field by the permanent magnet excitation magnetic field and armature magnetic field superposition. After the stacking make the motor armature magnetic field on the right side of the stator iron core air gap resultant magnetic field increases, and the left side is reduced, analytical calculate the magnetic flux density and finite element calculation of magnetic flux density is still edge effect and nonlinear changes the shape of the core which will be significant.

The Application and the Future. Linear oscillation motor for linear compressor drives according to its vibration components can be divided into moving coil, moving iron type, moving magnet type and moving magnet type four kinds. With the successful development of high performance permanent magnets, moving magnet the linear oscillatory motor with its simple structure, light quality and electromagnetic thrust density become a promising a structure. When type of refrigeration compressor was determined, are faced with the choice of compressor is the numbers. Determine the general principles of the compressor stations are: equipped with compressor stations should be less as far as possible, in order to simplify the system is easy to operation and management.

However, the total number of compressor should not be less than many, to ensure that the capacity of regulating when the load changes, and still have a sustainable maintenance refrigeration. Through linear oscillation motor direct drive the pistons, can greatly improve the efficiency of the motor and mechanical transmission efficiency, so as to achieve a substantial increase in refrigeration compressor performance coefficient and can effect comparing compared with traditional compressor, the purpose of the linear compressor not only save intermediate conversion mechanism crank shafts and so on, and the linear oscillation motor and compressor integration design, simple structure, easy encapsulation, high transmission efficiency, noise and vibration is small, easy to implement without oil lubrication, is particularly important, to adjust the volume of refrigeration, linear compressor can be used high performance frequency conversion control also can use low cost pressure variable stroke control way which will largely enhance the effectiveness of the motors. 


\section{Conclusion}

In this paper, we conduct research on the structure of linear oscillation motor and the applications on piston type refrigeration compressor. Linear oscillation motor mainly use electricity to produce high frequency reciprocating linear motion, push the work of working parts. While traditional reciprocating linear motion mainly through rotating motor plus Bi crank connecting rod mechanism, CAM mechanism and eccentric institutions such as a set of intermediate transformation mechanism, this linear motion technology mature, performance at the long working life. For winning line oscillation motor if the most typical application areas abundant piston type refrigeration compressor. Conventional piston compressor is driven by rotary motor, through the crank connecting rod mechanism such as mechanical drive system of the motor rotation into the reciprocating linear motion of the piston. Our research theoretically analyzes the corresponding issues combined with the literature review. In the future, we have scheduled to conduct more research to optimize the method.

\section{References}

[1] Xiong, C., Wang, L. Y., Zheng, W. P., Yang, K. X., Xiao-Ping, Q. U., \& Yi-Non, W. U. (2012). Power loss analysis of two kinds of moving-magnet linear oscillation motor. Small \& Special.

[2] Wang Y F, Gao G S. A Finite Element Analysis of Axisymmetric Magnetic Field of Linear Oscillation Motor with Various Excitation[J]. Journal of Liaoning Technical University, 2001.

[3] Franck,02Aloyse02J.02P, Nowak,02Maik. Non - Linear Oscillation Testing with a Separate Motor Transducer Rheometer[J]. Aip Conference Proceedings, 2008, 1027:1138-1140. 